\title{
GDF11/BMP11 as a novel tumor marker for liver cancer
}

\author{
YONG-HUI ZHANG ${ }^{1,2}$, LIAN-HONG PAN ${ }^{2}$, YI PANG ${ }^{2}$, JIN-XIN YANG ${ }^{2}$, MENG-JIA LV ${ }^{2}$, FENG LIU ${ }^{2}$, \\ XUE-FENG QU ${ }^{3}$, XIN-XIN CHEN ${ }^{2}$, HUA-JUN GONG ${ }^{2}$, DAN LIU ${ }^{1,2}$ and YONG WEI ${ }^{4}$ \\ ${ }^{1}$ Department of Basic Medical Science, Chongqing Three Gorges Medical College; \\ ${ }^{2}$ Chongqing Engineering Research Center of Antitumor Natural Drugs, Chongqing 404120; \\ ${ }^{3}$ Department of Nutrition and Food Hygiene, Zhejiang Academy of Medical Sciences, Hangzhou, Zhejiang 310013; \\ ${ }^{4}$ Key Laboratory of Intelligent Information Processing and Control, College of Electronic and Information Engineering, \\ Chongqing Three Gorges University, Chongqing 404110, P.R. China
}

Received July 13, 2017; Accepted December 8, 2017

DOI: $10.3892 /$ etm.2018.5861

\begin{abstract}
Growth differentiation factor 11 (GDF11), also known as bone morphogenetic protein 11, a member of the transforming growth factor- $\beta$ superfamily, has been reported to be involved in colorectal cancer. However, the roles of GDF11 in Chinese patients with liver cancer and the underlying mechanisms have remained elusive. The present study assessed the expression of GDF11 in 10 paired samples of cancerous and normal tissues from Chinese liver cancer patients. The results indicated that the expression of GDF11 was significantly lower in cancerous tissues than in normal tissues. In vitro, the expression of GDF11 was reduced in a panel of liver cancer cell lines compared with that in a normal liver cell line at the mRNA and protein level. Treatment with GDF11 reduced the viability of HepG2 for up to $72 \mathrm{~h}$ and GDF11 treatment reduced the viability of SMMC-7721 after 48 and 72 h. Furthermore, GDF11 activated Smad2/3 signaling in HepG2 cells. In conclusion, GDF11 has a tumor suppressor role in liver cancer, exerts its effects through Smad2/3 signaling and may serve as a novel tumor marker in liver cancer diagnosis.
\end{abstract}

\section{Introduction}

Liver cancer, also known as hepatic cancer, is a common malignant tumor that represents a severe threat to human

Correspondence to: Professor Dan Liu, Department of Basic Medical Science, Chongqing Three Gorges Medical College, 366 Tianxing Road, Wanzhou, Chongqing 404120, P.R. China

E-mail: 1036726924@qq.com

Dr Yong Wei, Key Laboratory of Intelligent Information Processing and Control, College of Electronic and Information Engineering, Chongqing Three Gorges University, 666 Tianxing Road, Wanzhou, Chongqing 404110, P.R. China

E-mail: weiyong198747@163.com

Key words: bone morphogenetic protein 11, growth differentiation factor 11, liver cancer, tumor marker, Smad2/3 health. According to the latest epidemiological study from the American Cancer Society, liver cancer is the fifth leading cause of cancer-associated mortality in men and the eighth leading cause in women. Furthermore, the death rate due to liver cancer is increasing (1). In China, liver cancer has the second highest mortality rate of all cancers in men and the fourth highest in women. Between 2000 and 2011, an increasing trend in the incidence and death rates of liver cancers has been observed (2). Therefore, it is an urgent issue to investigate the underlying molecular mechanisms to develop novel drugs and identify more sensitive tumor markers for liver cancer. However, knowledge of the molecular mechanisms associated with liver cancer remains insufficient and further elucidation thereof may improve the early diagnosis and prediction of liver cancer patient prognosis as an urgent issue. Numerous studies have assessed tumor markers of liver cancer. At present, $\alpha$-fetoprotein (AFP) is the most widely used tumor marker for the early detection of liver cancer (3). Besides AFP, AFP-L3 and squamous cell carcinoma antigen are used for the early diagnosis of liver cancer and heat shock protein 70, TGF- $\beta$ and microRNA-500/29/112 are used as prognostic markers for liver cancer (4). However, the specificity and sensitivity of these tumor markers are not satisfactory. Therefore, the present study assessed whether GDF11 may be a novel tumor marker in liver cancer.

Growth differentiation factor 11 (GDF11), also known as bone morphogenetic protein 11 (BMP11), was first cloned and characterized as a member of the BMP/transforming growth factor $\beta$ (TGF- $\beta$ ) superfamily (5). The GDF11 gene was mapped to human chromosome $12 q 13.2$ by aligning the GDF11 sequence (GenBank ID AF100907) with the genomic sequence (GRCh38). It encodes a 407-amino acid protein with a signaling sequence for secretion, an RXXR proteolytic processing site and a region at the $\mathrm{C}$-terminal end containing a highly conserved pattern of cysteine residues (6). GDF11 protein, cleaved by pro-protein convertase subtilisin/kexin type 5 (PCSK5), forms a non-covalent latent complex, which contains an inhibitory pro-domain at the N-terminus and two disulfide-linked active domains at the C-terminus $(7,8)$. For the GDF11-induced signal pathway, GDF11 binds to activin receptor type IIB (ACTRIIB) and subsequently, the complex recruits the activin receptor-like kinases (ALKs) ALK4, ALK5 
and ALK7 to activate the receptor Smad (R-Smad) pathway involving Smad2/3, which associate with Smad4. The Smad protein complex then translocates into the nucleus to positively or negatively control gene expression $(9,10)$.

GDF11 not only contributes to embryonic development and histogenesis, but also has a role in metabolic disorders, cardiovascular diseases and ageing (11-14). GDF11 is expressed in the liver and has an inhibitory role in liver development. It was reported that overexpression of GDF11 in embryos by mRNA microinjection produced a small liver phenotype. The mechanism possibly comprises the suppression of hepatocyte proliferation (15). The observation that GDF11 suppresses liver development raises the question whether GDF11 has a similar function in liver cancer. In the field of cancer research, various studies have investigated the role of GDF11, indicating that it is probably involved in colorectal cancer, breast cancer and leiomyoma uteri $(11,16-18)$. However, the role of GDF11 in liver cancer has been rarely reported, which was therefore investigated in the present study.

In the present study, the levels of GDF11 in liver cancer patients and liver cancer cell lines compared with those in matched normal liver tissue or cells were assessed in order to identify whether the level of GDF11 changes with the development of liver cancer. Due to the high homology between the active domain of GDF11 and myostatin, the expression was assessed at the mRNA level (19). As GDF11 belongs to the TGF- $\beta$ /BMP superfamily, the canonical signaling associated with the latter should be the basis of the mechanism of action of GDF11. Therefore, the effects of GDF11 on Smad signaling in the human hepatoma cancer cell line HepG2 were identified and the effect of GDF11 on the viability of HepG2 cells was then studied. The present study was the first to investigate the expression and role of GDF11 in liver cancer patients.

\section{Materials and methods}

Reagents and cell lines. The complementary (c)DNA cohort of 10 pairs of human malignant liver cancer tissues and their corresponding adjacent non-cancerous liver tissues was purchased from Shanghai Outdo Biotech Co., Ltd (Shanghai, China; cat. no. cDNA-HLivH30PG01). The samples were derived from Chinese patients with hepatocellular carcinoma and were characterized by a pathological grade of II-III, an age range from 51 to 72 years, no distant metastasis, no perineural invasion and an American Joint Committee on Cancer clinical stage of 1-2 (Table I). Fetal bovine serum (FBS) was purchased from Natocor-Industria Biológica (Córdoba, Argentina). Recombinant human/mouse/rat GDF-11 was obtained from PeproTech (Rocky Hill, NJ, USA; cat. no. 120-11). Anti-phosphorylated (p)-Smad3 (Ser423/425), anti-Smad3, anti-p-Smad2 (Ser465/467), anti-Smad2 and anti-Smad2/3 were obtained from Cell Signaling Technology (Danvers, MA, USA; cat. no. 12747). Anti-GDF11 antibody was purchased from Abcam (Cambridge, UK; cat. no. ab71347). Anti- $\beta$-actin was obtained from Zhongshan Goldenbridge Bio (Beijing, China; cat. no. TA-09). The SYBR-Green RT-PCR kit was obtained from Takara Bio, Inc. (Otsu, Japan). Primers were designed and synthesized by Shanghai Sangon Biotech. Co., Ltd. (Shanghai, China). Smad3 inhibitor SIS3 was purchased from Santa Cruz Biotechnology, Inc. (Dallas, TX, USA).
Cell culture. The L-02,HepG2 and SMMC-7721 cell lines were provided by the Chongqing Engineering Research Center of Antitumor Natural Drugs (Chongqing, China) and purchased from Shanghai Zhong Qiao Xin Zhou Biotechnology Co., Ltd. (Shanghai, China). HepG2 is known to be misidentified as a hepatoblastoma cell line (20). Cells were cultured in RPMI 1640 medium (Hyclone; GE Healthcare, Little Chalfont, UK) supplemented with 10\% FBS in a humidified incubator containing $5 \% \mathrm{CO}_{2}$. Recombinant GDF11 pure protein used for treating the cells was purchased from PeproTech Inc. and for the preparation of a stock solution, $20 \mu \mathrm{g}$ GDF11 was dissolved in $40 \mu 1$ sterile ultra-pure water according to the manufacturer's protocol. Recombinant GDF11 pure protein was utilized according to methods described in previous studies $(13,14)$. Cells in the control group were treated with the same volume of sterile ultra-pure water.

Reverse transcription-quantitative polymerase chain reaction $(R T-q P C R)$. The cDNA microarray is a novel type of cDNA cohort to detect the expression of a target gene through RT-qPCR. cDNA samples were derived from 10 pairs of human malignant liver cancer tissues and their corresponding adjacent non-cancerous liver tissues with complete clinical information. For the cell lines, total RNA was extracted from the normal liver cell line L-02 and liver cancer cell lines (HepG2 and SMMC-7721) with TRIzol reagent (Thermo Fisher Scientific, Inc., Waltham, MA, USA). The total RNA was then converted into cDNA with the RevertAid First Strand cDNA Synthesis Kit (Thermo Fisher Scientific, Inc.) according to the manufacturer's instructions. Real-time qPCR was performed in a Roche LightCycler 480II (Roche Diagnostics, Basel, Switzerland) with the SYBR Green PCR master mix (Takara Bio Inc.). $\beta$-actin was used as an internal reference. The specific primers used to detect GDF11 were as follows: Forward, 5'-GCCATCAACACCACTCACATT-3' and reverse, 5'-CCAATCCCTACTCTGCCAAG-3'. The specific primers used for $\beta$-actin were as follows: Forward, 5'-GAAGAG CTACGAGCTGCCTGA-3' and reverse, 5'-CAGACAGCA CTGTGTTGGCG-3'. The thermocycling conditions were as follows: Initial denaturation at $95^{\circ} \mathrm{C}$ for $30 \mathrm{sec}$, followed by 40 cycles of denaturation at $95^{\circ} \mathrm{C}$ for $5 \mathrm{sec}$ and an annealing/elongation step at $60^{\circ} \mathrm{C}$ for $30 \mathrm{sec}$. Experiments were performed in triplicate and the values were normalized to $\beta$-actin. Relative expression was analyzed via the $2^{-\Delta \Delta C q}$ method (21).

Western blot analysis. Western blot analysis was performed as described in previous studies by our group $(14,22)$. Cells were lysed with radioimmunoprecipitation assay buffer (Beyotime Institute of Biotechnology, Haimen, China) and centrifuged at $12,000 \mathrm{x} \mathrm{g}$ for $15 \mathrm{~min}$ at $4^{\circ} \mathrm{C}$. The supernatants were collected and the protein concentrations were determined with a bicinchoninic acid assay kit (Beyotime Institute of Biotechnology). The protein (50 $\mu \mathrm{g} / \mathrm{lane})$ was subjected to $10 \%$ SDS-PAGE and transferred to polyvinylidene difluoride membranes (Beyotime Institute of Biotechnology). After blocking with 5\% nonfat milk at $4^{\circ} \mathrm{C}$ for $2 \mathrm{~h}$, the membranes were probed with primary antibodies against the analyte proteins stated above (1:500 dilution) and $\beta$-actin (1:1,000 dilution) at $4^{\circ} \mathrm{C}$ overnight. Following three washes with PBS, the membrane was incubated with mouse anti-rabbit immunoglobulin $\mathrm{G}$ conjugated 
Table I. Clinicopathological variables of 10 liver cancer patients.

\begin{tabular}{lc}
\hline Variable & N/value \\
\hline Age (years \pm SEM) & $57.0 \pm 7.77$ \\
Gender & \\
Male & 9 \\
Female & 1 \\
Pathological grade & II \\
Tumor site & \\
Right liver & 7 \\
Left liver & 3 \\
Nerve invasion & \\
Absent & 6 \\
Unknown & 4 \\
Vascular invasion & \\
Absent & 4 \\
Present & 3 \\
Unknown & 3 \\
AJCC stage & \\
1 & 4 \\
2 & 3 \\
Unknown & 3 \\
\hline
\end{tabular}

SEM, standard error of the mean; AJCC, American Joint Committee on Cancer.

to horseradish peroxidase (1:1,000 dilution; Cell Signaling Technology, Inc.; cat. no. 7074) at room temperature for $1 \mathrm{~h}$. A chemiluminescence kit (Bio-Rad Laboratories, Inc., Hercules, CA, USA) was then used to perform chemiluminescent detection, according to the manufacturer's instructions. Western blot bands were quantified by using the ChemiDoc ${ }^{\mathrm{TM}}$ Touch Imaging System (Bio-Rad Laboratories, Inc.) and Image Lab ${ }^{\mathrm{TM}}$ Touch Software (version 1.2; Bio-Rad Laboratories, Inc.).

MTT assay. Cell viability was assessed using an MTT assay (Biosharp, Shanghai, China), which measures mitochondrial succinate dehydrogenase activity in living cells. The assay is dependent on the ability of viable cells to metabolize a water-soluble tetrazolium salt into a water-insoluble formazan product. Cells were trypsinized and seeded in a 96-well plate. After adherence, complete medium was replaced with basal medium for $12 \mathrm{~h}$. Following an incubation of 24, 48 and $72 \mathrm{~h}$ at $37^{\circ} \mathrm{C}$ with the respective reagents, a total of $100 \mu \mathrm{l}$ RPMI-1640 medium (Hyclone; GE Healthcare) containing $0.5 \mathrm{~g} / \mathrm{l}$ MTT was added to each well, followed by $4 \mathrm{~h}$ of incubation. Subsequently, the medium was removed by aspiration, followed by addition of $50 \mu \mathrm{l}$ dimethyl sulfoxide. Following incubation at $37^{\circ} \mathrm{C}$ for $10 \mathrm{~min}$, the absorbance of each well at $490 \mathrm{~nm}$ was measured using a microplate reader.

Statistical analysis. Values are expressed as the mean \pm standard error of the mean. Significance was determined by using Student's t-test or one-way analysis of variance, followed by the
Holm-Sidak test. Statistical analysis of the data was performed using GraphPad Prism 5 software (GraphPad Software, Inc., La Jolla, CA, USA). $\mathrm{P}<0.05$ was considered to indicate a statistically significant difference.

\section{Results}

GDF11 is downregulated in liver cancer tissues and cell lines. A cDNA microarray of 10 paired cancerous and normal tissue samples from liver cancer patients was used to detect the mRNA levels of GDF11. The results indicated that the expression of GDF11 in human malignant liver cancer tissues at the mRNA level was decreased compared with that in the corresponding adjacent non-cancerous liver tissues $(\mathrm{P}<0.05$; Fig. 1A). To verify the results, the database Oncomine was consulted (www.oncomine.com) and the search indicated that GDF11 expression was also declined according the database information (Fig. 1B). As GDF11 mRNA levels were lower in the clinical liver cancer tissue samples compared with those in the normal adjacent tissues, GDF11 levels were also assessed in liver cancer cell lines and a normal liver cell line to confirm this trend. In vitro, the level of GDF11 in the liver cancer cell lines SMMC-7721 and HepG2 was compared with that in the normal liver cancer cell line L-O2 at the mRNA and protein level. According to Fig. 1C and D, GDF11 mRNA and protein levels were significantly decreased in liver cancer cell lines. These results indicate that GDF11 may serve as a tumor suppressor in liver cancer. Subsequently, the mechanisms of GDF11 in liver cancer were assessed in in vitro experiment.

GDF11 activates Smad2/3 signaling in HepG2 cells and decreases the viability of HepG2 cells. Firstly, we verified GDF11 expression was indeed increased following the treatment of cells with recombinant GDF11. HepG2 and SMMC-7721 cells were treated with GDF11 (50 or $100 \mathrm{ng} / \mathrm{ml}$ ), which significantly increased the levels of GDF11 (Fig. 2A). Activation of Smad2/3 is a typical effect of TGF- $\beta$ members (23); therefore, it was then detected whether GDF11 activates Smad2/3 signaling in HepG2 cells. The hepatoma cell line HepG2 was treated with GDF11 (50 and $100 \mathrm{ng} / \mathrm{ml}$ ) for $15 \mathrm{~min}$ or $1 \mathrm{~h}$. The results indicated that GDF11 significantly increased the levels of p-Smad2 and p-Smad3 compared with those in the control group (Fig. 2B). Furthermore, Smad3 activation by GDF11 was inhibited by Smad3 inhibitor SIS3 (5 $\mu \mathrm{M}$; Fig. 2C). Smad2 and Smad3 are known to participate in tumor-associated processes, Smad2 mutations have been detected in numerous cancer types and Smad2, as a tumor suppressor, mediates the TGF- $\beta$-induced tumor-suppressive function (24-26). Similarly, Smad3 also has an inhibitory function in tumors by suppressing cell proliferation and promoting apoptosis (27). Therefore, GDF11 may suppress processes associated with liver cancer by activating Smad2/3 signaling. In addition, In order to verify whether GDF11 acts as a tumor suppressor in vitro, HepG2 and SMMC-7721 cells were treated with GDF11 for different duration (24, 48 and $72 \mathrm{~h}$ ) at the concentration of 50 or $100 \mathrm{ng} / \mathrm{ml}$. According to the results, GDF11 did not affect the cell viability for 24 and $48 \mathrm{~h}$, but the viability of HepG2 cells was significantly decreased after treatment for $72 \mathrm{~h}$ (Fig. 3A). For the SMMC-7721 cell line, a similar result was obtained, namely that GDF11 decreased cell viability after treatment of 48 and $72 \mathrm{~h}$ (Fig. 3B). These results 
A

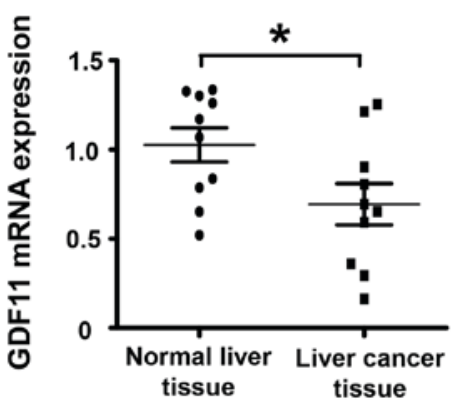

C

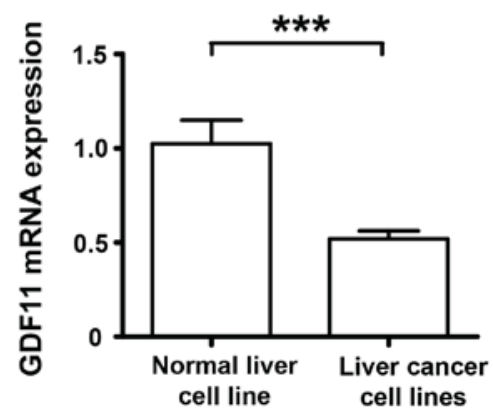

B

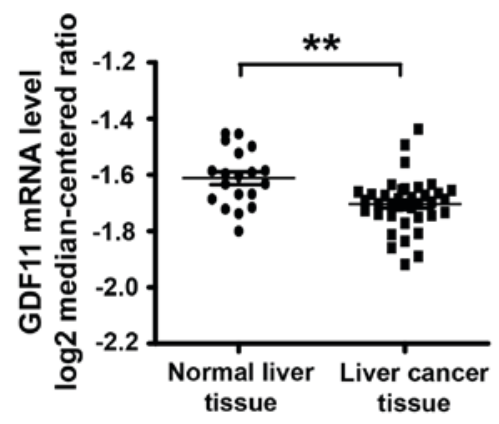

D

L-O2 L-O2 HepG2 SMMC-7721

GDF11

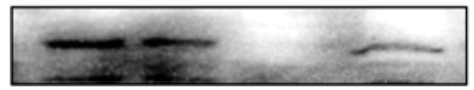

$\beta$-actin

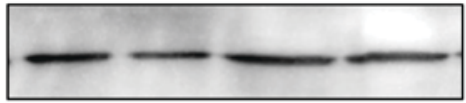

Figure 1. The expression of GDF11 is decreased in liver cancer tissue at the mRNA level and is downregulated in liver cancer cells. (A) GDF11 mRNA expression in 10 paired samples of cancerous and normal liver tissue in Chinese patients with liver cancer. Cancer tissues had significantly lower GDF11 mRNA expression than normal tissues. (B) Oncomine data indicating GDF11 expression in normal vs. cancerous liver tissues. (C) GDF11 mRNA expression in a normal liver cell line and liver cancer cell lines (HepG2 and SMMC-7721).(D) GDF11 protein expression in a normal liver cell line and liver cancer cell lines (HepG2 and SMMC-7721). Data are presented as the mean \pm standard error of the mean. ${ }^{*} \mathrm{P}<0.05$ vs. normal tissues $(\mathrm{n}=10)$; ${ }^{* *} \mathrm{P}<0.01 \mathrm{vs}$. normal tissues $(\mathrm{n}=19)$;

${ }_{* * * *}^{*}<0.001$ vs. normal liver cell line. GDF11, growth differentiation factor 11.

A

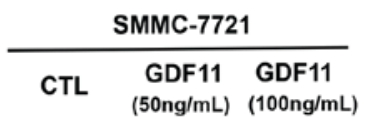

GDF11

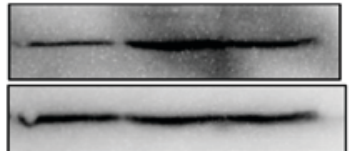

$\beta$-actin

B

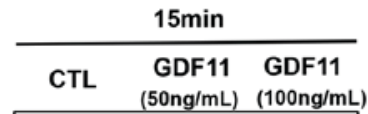

\section{(p)}

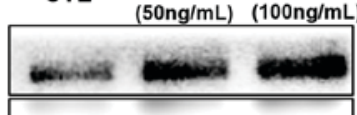

p-Smad2

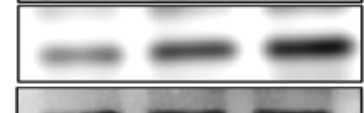

Smad2/3

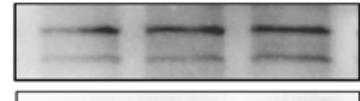

$\beta$-actin

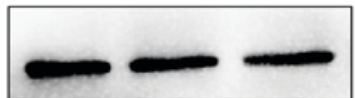

C

C

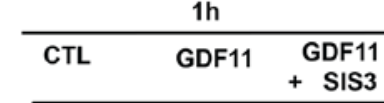

p-Smad3

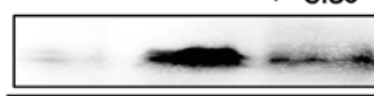

$\beta$-actin
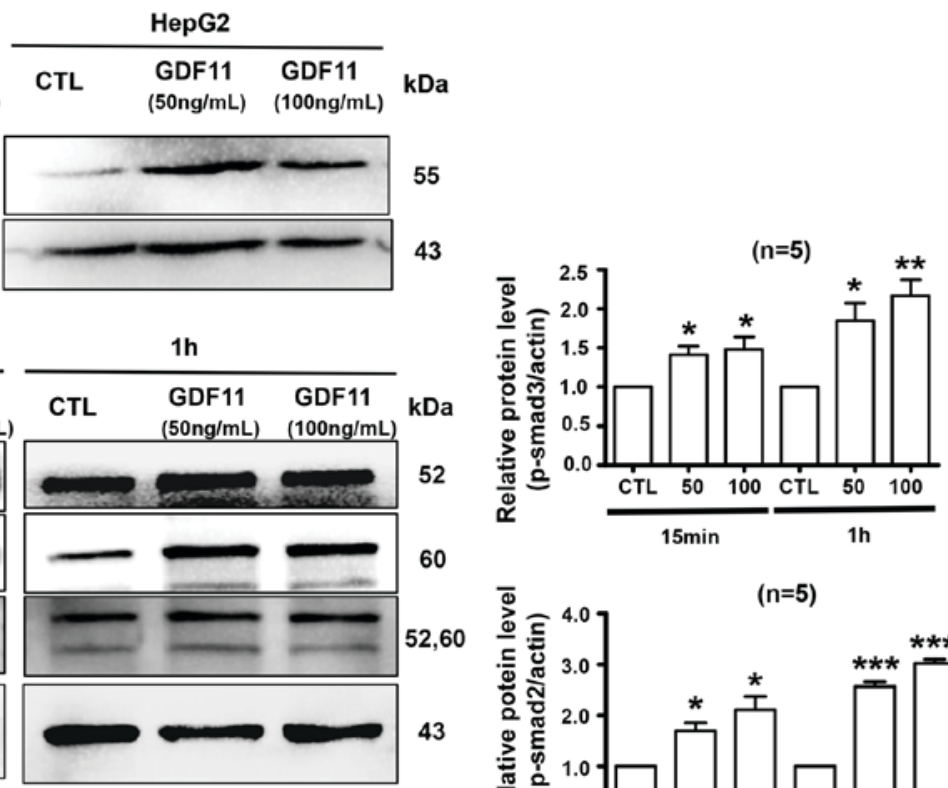

kDa

52

43

Figure 2. GDF11 increases the level of p-Smad2/3 protein in HepG2 cells. (A) Recombinant GDF11 increased the protein expression of GDF11 in SMMC-7721 and HepG2 cells. Cells in the control group were treated with the same volume ( $5 \mu 1$ per cell culture flask) of sterile ultra-pure water. (B) HepG2 cells treated with GDF11 (50 and $100 \mathrm{ng} / \mathrm{ml}$ ) for $15 \mathrm{~min}$ and $1 \mathrm{~h}$. The phosphorylation of Smad2/3 was increased after GDF11 treatment in HepG2 cells. (C) The activation of Smad3 by GDF11 was attenuated in the presence of the Smad3 inhibitor SIS3 $(5 \mu \mathrm{M})$. $\beta$-actin was used as an internal reference. ${ }^{*} \mathrm{P}<0.05,{ }^{* * *} \mathrm{P}<0.01$ and ${ }^{* * * *} \mathrm{P}<0.001$ vs. CTL group $(\mathrm{n}=5)$. CTL group is normalized to 1. GDF11, growth differentiation factor 11; CTL, control; p-Smad3, phosphorylated Smad3. 
demonstrate the tumor suppressive effect of GDF11 in liver cancer.

\section{Discussion}

Exploring novel tumor markers is important for improving the diagnosis and treatment of liver cancer. GDF11 was reported to be expressed in the liver and to have a regulatory function in liver development (13). Overexpression of GDF11 by mRNA microinjection in an embryonic animal model led to a small liver phenotype in a dose-dependent manner, indicating that GDF11 suppressed the growth/expansion phase of liver development, possibly by acting as an inhibitor of cell proliferation. Based on this result, it may be hypothesized that GDF11 has a similar role in liver cancer, namely that of a tumor suppressor. Several studies have suggested that GDF11 is involved in cancer $(11,17,18)$. A study by Yokoe et al (11) indicated that GDF11 was positively associated with processes of colorectal cancer. They identified that the expression of GDF11 mRNA was increased in colorectal cancer tissues and that patients with high GDF11 expression had a poorer prognosis. However, histone deacetylases (HDACs), key transcriptional regulators that inhibit GDF11 gene expression, were proved to promote tumor growth in animals $(28,29)$. Several HDAC inhibitors, including vorinostat (SAHA), romidepsin, belinostat and panobinostat, have been approved by the USA food and drug administration to treat cancer (29). The mechanisms of action of HDAC inhibitors may include inhibition of abnormal cell growth by inactivation of HDAC3 and activation of GDF11 expression (14). The function of GDF11 in various cancer types remains controversial. Therefore, the present study investigated the possible role of GDF11 as a tumor promoter or inhibitor in liver cancer. First, the mRNA expression of GDF11 in cancerous liver tissues was compared with that in normal liver tissue. The mRNA and protein levels of GDF11 in a normal liver cell line and liver cancer cell lines were also assessed. In a subsequent in vitro experiment, the effect of GDF11 on the viability of the liver cancer cell lines HepG2 and SMMC-7721 was observed. The results indicated that GDF11 expression was decreased in liver cancer tissues compared with that in normal liver tissues, which was in accordance with the result retrieved from the Oncomine database. These results were consistent with those obtained with the cell lines results, namely that GDF11 was downregulated in liver cancer cell lines. In vitro, treatment with recombinant GDF11 for up to $72 \mathrm{~h}$ led to a reduction of cell viability in HepG2 and SMMC-7721 cells, possibly by activating Smad2/3 signaling. It was previously demonstrated that GDF11 treatment leads to an upregulation of p-Smad3 in multiple cell types, including pluripotent stem cell-derived cardiomyocytes, human skeletal muscle-derived cells and human umbilical vein endothelial cells, to regulate cell proliferation and muscle regeneration $(10,13,14,30)$, which is consistent with the present results. Following activation of Smad2/3 by phosphorylation through GDF11, p-Smad2/3 forms a complex with Smad4, which then translocates to the nucleus to regulate cell function by affecting the expression of associated genes. The probable signaling pathway is summarized in Fig. 4.

In the present study, 10 pairs of human malignant liver cancer tissues and their corresponding adjacent non-cancerous liver tissues were assessed in a cDNA microarray. The samples are characterized by pathological grading II-III, age ranging
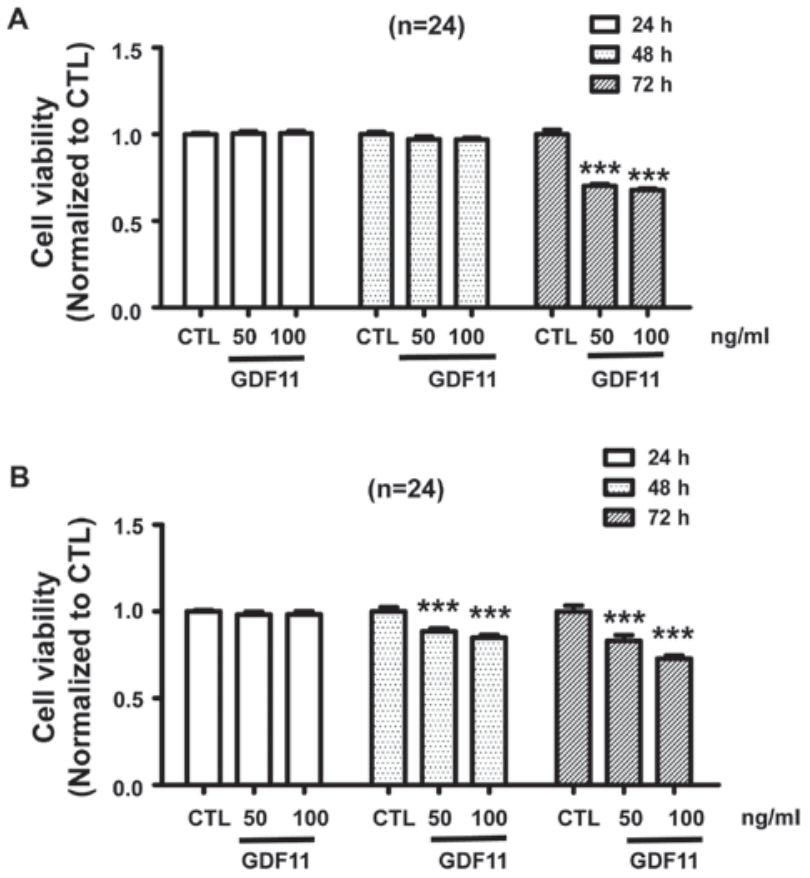

Figure 3. Treatment with GDF11 reduced the viability of HepG2 for up to $72 \mathrm{~h}$ and GDF11 treatment reduced the viability of SMMC-7721 after 48 and $72 \mathrm{~h}$. (A) HepG2 cells treated with GDF11 (50 and $100 \mathrm{ng} / \mathrm{ml}$ ) for 24, 48 and $72 \mathrm{~h}$. The cell viability was decreased following $72 \mathrm{~h}$ of incubation, while it remained unchanged at 24 and $48 \mathrm{~h}$. (B) SMMC-7721 cells treated with GDF11 (50 and $100 \mathrm{ng} / \mathrm{ml}$ ) for 24,48 and $72 \mathrm{~h}$. The cell viability was decreased following 48 and $72 \mathrm{~h}$ of treatment, while it remained unchanged for $24 \mathrm{~h} .{ }^{* * * *} \mathrm{P}<0.001$ vs. CTL group $(\mathrm{n}=24)$. GDF11, growth differentiation factor 11; CTL, control.

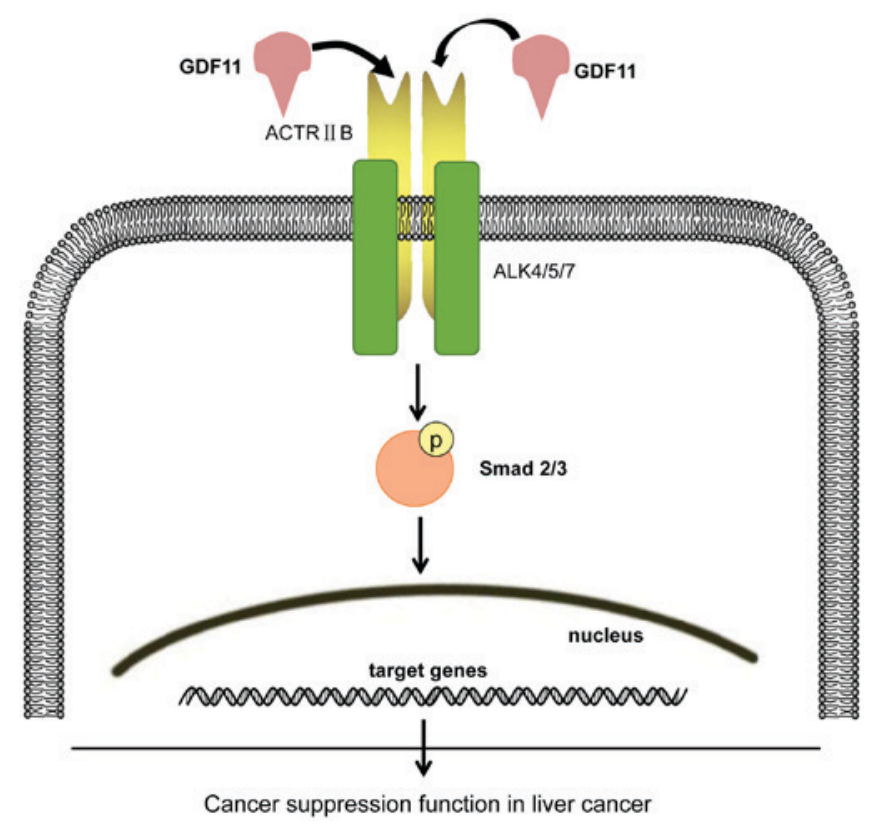

Figure 4. Schematic of GDF11 signaling in human liver cancer. GDF11 first binds to ActRIIB to recruit the type I ALK receptors ALK4, ALK5 and ALK7. The downstream pathways of GDF11 binding to its receptors include Smad and non-Smad pathways. The Smad pathway is the canonical signaling for transforming growth factor- $\beta$ superfamily members. GDF11 first activates receptor Smads, including Smad2/3 and Smad1/5/8 signals. After phosphorylation, the receptor Smads are released from the receptor and recruit the common Smad4 to form a complex, which then migrates into the nucleus and activates the transcription of specific target genes that have a role in liver cancer. GDF11, growth differentiation factor 11; p, phosphate; ActRIIB, activin receptor type IIB; ALK, activin receptor-like kinase. 
from 51 to 72 years, no distant metastasis, no perineural invasion and American Joint Committee on Cancer clinical stage 1-2, as presented in Table I. GDF11 mRNA expression was declined in these liver cancer tissues compared with that in adjacent non-cancerous liver tissues. However, Yokoe et al (11) reported that the expression of GDF11 mRNA was increased in colorectal cancer tissues and that patients with high GDF11 expression in their tumors had a higher frequency of lymph node metastasis and more cancer-associated mortalities. This divergent result may be due to the dual role of TGF- $\beta$ members. In early carcinomas, TGF- $\beta$ signaling pathways exert tumor suppressor effects and as tumors develop and progress, the role of TGF- $\beta$ signaling switches to promote cancer metastasis (31). Therefore, further investigation is required to explore the exact functional role of GDF11 regarding the regulation of cell viability, death and proliferation of liver cancer cells and the underlying molecular mechanisms, in addition to the study of GDF11 expressional changes in late-stage liver carcinomas.

In conclusion, the present study indicated that GDF11 reduced the viability of liver cancer cells, probably and at least in part via activation of Smad2/3 signaling. GDF11 mRNA expression was downregulated in liver cancer tissues compared with that in the corresponding normal tissues as indicated by a cDNA microarray and a search of the database Oncomine, which provided similar results to those obtained with liver cancer cell lines. These results indicate that GDF11 is a novel candidate for a tumor marker in patients with liver cancer.

\section{Acknowledgements}

This study was supported by the Science and Technology Research Projects of Chongqing Education Commission (grant nos. KJ1725391 and KJ1710238), the Natural Science Research Projects of Chongqing Three Gorges Medical College (grant no. 2016xmpxz02) and the National Natural Science Foundation of China (grant no. 61705025).

\section{References}

1. Siegel RL, Miller KD and Jemal A: Cancer statistics, 2016. CA Cancer J Clin 66: 7-30, 2016.

2. Chen W, Zheng R, Baade PD, Zhang S, Zeng H, Bray F, Jemal A, Yu XQ and He J: Cancer statistics in China, 2015. CA Cancer J Clin 66: 115-132, 2016.

3. Baig JA, Alam JM, Mahmood SR, Baig M, Shaheen R, Sultana I and Waheed A: Hepatocellular carcinoma (HCC) and diagnostic significance of A-fetoprotein (AFP). J Ayub Med Coll Abbottabad 21: 72-75, 2009.

4. Zhao YJ, Ju Q and Li GC: Tumor markers for hepatocellular carcinoma. Mol Clin Oncol 1: 593-598, 2013.

5. Nakashima M, Toyono T, Akamine A and Joyner A: Expression of growth/differentiation factor 11 , a new member of the BMP/TGFbeta superfamily during mouse embryogenesis. Mech Dev 80: 185-189, 1999.

6. Gamer LW, Wolfman NM, Celeste AJ, Hattersley G, Hewick R and Rosen V: A novel BMP expressed in developing mouse limb, spinal cord, and tail bud is a potent mesoderm inducer in Xenopus embryos. Dev Biol 208: 222-232, 1999.

7. Essalmani R, Zaid A, Marcinkiewicz J, Chamberland A, Pasquato A, Seidah NG and Prat A: In vivo functions of the proprotein convertase PC5/6 during mouse development: Gdf11 is a likely substrate. Proc Natl Acad Sci USA 105: 5750-5755, 2008.

8. Tsuda T, Iwai N, Deguchi E, Kimura O, Ono S, Furukawa T, Sasaki Y, Fumino S and Kubota Y: PCSK5 and GDF11 expression in the hindgut region of mouse embryos with anorectal malformations. Eur J Pediatr Surg 21: 238-241, 2011.
9. Rochette L, Zeller M, Cottin Y and Vergely C: Growth and differentiation factor 11 (GDF11): Functions in the regulation of erythropoiesis and cardiac regeneration. Pharmacol Ther 156: 26-33, 2015.

10. Camici GG, Savarese G, Akhmedov A and Lüscher TF: Molecular mechanism of endothelial and vascular aging: Implications for cardiovascular disease. Eur Heart J 36: 3392-3403, 2015.

11. Yokoe T, Ohmachi T, Inoue H, Mimori K, Tanaka F, Kusunoki M and Mori M: Clinical significance of growth differentiation factor 11 in colorectal cancer. Int J Oncol 31: 1097-1101, 2007.

12. Smith SC, Zhang X, Zhang X, Gross P, Starosta T, Mohsin S, Franti M, Gupta P, Hayes D, Myzithras M, et al: GDF11 does not rescue aging-related pathological hypertrophy. Circ Res 117: 926-932, 2015.

13. Loffredo FS, Steinhauser ML, Jay SM, Gannon J, Pancoast JR, Yalamanchi P, Sinha M, Dall'Osso C, Khong D, Shadrach JL, et al: Growth differentiation factor 11 is a circulating factor that reverses age-related cardiac hypertrophy. Cell 153: 828-839, 2013.

14. Zhang YH, Cheng F, Du XT, Gao JL, Xiao XL, Li N, Li SL and Dong de L: GDF11/BMP11 activates both Smad1/5/8 and Smad2/3 signals but shows no significant effect on proliferation and migration of human umbilical vein endothelial cells. Oncotarget 7: 11063-11074, 2016.

15. Farooq M, SuLochana KN, Pan X, To J, Sheng D, Gong Z and Ge R: Histone deacetylase3(HDAC3) is specifically required for liver development in zebrafish. Dev Biol 317: 336-353, 2008.

16. Bajikar SS, Wang CC, Borten MA, Pereira EJ, Atkins KA and Janes KA: Tumor-suppressor inactivation of GDF11 occurs by precursor sequestration in triple-negative breast cancer. Dev Cell 43: 418-435, 2017.

17. Alvarez C, Aravena A, Tapia T, Rozenblum E, Solís L, Corvalán A, Camus M, Alvarez M, Munroe D, Maass A and Carvallo P: Different Array CGH profiles within hereditary breast cancer tumors associated to BRCA1 expression and overall survival. BMC Cancer 16: 219, 2016.

18. Auguściak-Duma A and Sieroń AL: Molecular characteristics of leiomyoma uteri based on selected compounds of the extracellular matrix. Postepy Hig Med Dosw (Online) 62: 148-165, 2008 (In Polish).

19. McNally EM: Questions and answers about myostatin, GDF11, and the aging heart. Circ Res 118: 6-8, 2016.

20. López-Terrada D, Cheung SW, Finegold MJ and Knowles BB Hep G2 is a hepatoblastoma-derived cell line. Hum Pathol 40: $1512-1515,2009$.

21. Livak KJ and Schmittgen TD: Analysis of relative gene expression data using real-time quantitative PCR and the 2(-Delta Delta C(T)) method. Methods 25: 402-408, 2001

22. Sheng Y, Sun B, Guo WT, Liu X, Wang YC, Xie X, Xiao XL, $\mathrm{Li} \mathrm{N}$ and Dong DL: [4-(6-(4-isopropoxyphenyl)pyrazolo(1,5-a) pyrimidin-3-yl)quinoline] is a novel inhibitor of autophagy. Br J Pharmacol 171: 4970-4980, 2014.

23. Dwivedi SK, McMeekin SD, Slaughter K and Bhattacharya R: Role of TGF- $\beta$ signaling in uterine carcinosarcoma. Oncotarget 6 : 14646-14655, 2015.

24. Levy L and Hill CS: Alterations in components of the TGF-beta superfamily signaling pathways in human cancer. Cytokine Growth Factor Rev 17: 41-58, 2006.

25. Prunier C, Ferrand N, Frottier B, Pessah M and Atfi A: Mechanism for mutational inactivation of the tumor suppressor Smad2. Mol Cell Biol 21: 3302-3313, 2001.

26. Yang J, Wahdan-Alaswad R and Danielpour D: Critical role of Smad2 in tumor suppression and transforming growth factor-beta-induced apoptosis of prostate epithelial cells. Cancer Res 69: 2185-2190, 2009.

27. Millet $\mathrm{C}$ and Zhang YE: Roles of Smad3 in TGF-beta signaling during carcinogenesis. Crit Rev Eukaryot Gene Expr 17: 281-293, 2007.

28. Ropero S and Esteller M: The role of histone deacetylases (HDACs) in human cancer. Mol Oncol 1: 19-25, 2007.

29. Li Y and Seto E: HDACs and HDAC inhibitors in cancer development and therapy. Cold Spring Harb Perspect Med 6: pii: a026831, 2016.

30. Egerman MA, Cadena SM, Gilbert JA, Meyer A, Nelson HN, Swalley SE, Mallozzi C, Jacobi C, Jennings LL, Clay I, et al: GDF11 increases with age and inhibits skeletal muscle regeneration. Cell Metab 22: 164-174, 2015.

31. Lebrun JJ: The dual role of TGF $\beta$ in human cancer: From tumor suppression to cancer metastasis. ISRN Mol Biol 2012: 381428, 2012. 